\title{
The monetary value of diets consumed by British adults: an exploration into sociodemographic differences in individual-level diet costs
}

\author{
Kate A Timmins ${ }^{1, *}$, Claire Hulme ${ }^{2}$ and Janet E Cade ${ }^{1}$ \\ 'Nutritional Epidemiology Group, School of Food Science \& Nutrition, University of Leeds, Leeds LS2 9JT, UK: \\ ${ }^{2}$ Academic Unit of Health Economics, Leeds Institute of Health Sciences, University of Leeds, Leeds, UK
}

Submitted 20 February 2013: Final revision received 16 September 2013: Accepted 23 September 2013: First published online 29 0ctober 2013

\begin{abstract}
Objective: To describe the diet costs of adults in the National Diet and Nutrition Study (NDNS) and explore patterns in costs according to sociodemographic indicators.

Design: Cross-sectional diet diary information was matched to a database of food prices to assign a cost to each food or non-alcoholic beverage consumed. Daily diet costs were calculated, as well as costs per $10 \mathrm{MJ}$ to improve comparability across differing energy requirements. Costs were compared between categories of sociodemographic variables and health behaviours. Multivariable regression assessed the effects of each variable on diet costs after adjustment.

Setting: The NDNS is a rolling dietary survey, recruiting a representative UK sample each year. The study features data from 2008-2010.

Subjects: Adults aged 19 years or over were included. The sample consisted of 1014 participants.

Results: The geometric mean daily diet cost was $£ 2 \cdot 89$ (95\% CI £2.81, £2.96). Energy intake and daily diet cost were strongly associated. The mean energyadjusted cost was $£ 4 \cdot 09$ (95\% CI £4·01, £4·18) per $10 \mathrm{MJ}$. Energy-adjusted costs differed significantly between many subgroups, including by sex and household income. Multivariable regression found significant effects of sex, qualifications and occupation (costs per $10 \mathrm{MJ}$ only), as well as equivalized household income, BMI and fruit and vegetable consumption on diet costs.

Conclusions: This is the first time that monetary costs have been applied to the diets of NDNS adults. The findings suggest that certain subgroups in the UK - for example those on lower incomes - consume diets of lower monetary value. Observed differences were mostly in the directions anticipated.
\end{abstract}

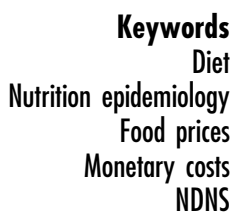

Food cost is an accepted determinant of dietary decision making ${ }^{(1-5)}$. Trends in the price of foods are thought to influence the selection of different types of foods, therefore having the potential to affect diet quality. For example, the falling real price of food, and in particular of energy-dense foods, is suggested to have encouraged the overconsumption of energy and could thus be implicated in global obesity trends ${ }^{(6,7)}$.

Measuring the monetary costs of diets, however, is not straightforward. The tracking of food prices at a national level or the collection of household-level food expenditure surveys is not uncommon (e.g. reference 8); however, no studies, to the authors' knowledge, have simultaneously collected individual-level expenditure and dietary consumption data. As a result, the cost of food actually consumed, as opposed to the cost of food purchased, has never been directly measured. Consequently, researchers must infer dietary consumption from purchasing data or, vice versa, infer costs from dietary assessment. In the field of public health nutrition, the latter method confers the advantage, making use of established dietary assessment techniques. For this reason, assigning a monetary cost to individual-level dietary data using a database of national average food prices is an increasingly popular $\operatorname{method}^{(6,9)}$.

Previous publications have employed this method to report the monetary value of the diets of American ${ }^{(9,10)}$,

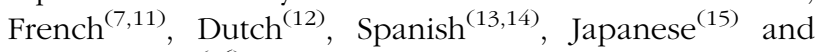
British female $^{(16)}$ populations, but none have done so in a representative UK sample.

The current study describes for the first time the monetary values of adults' diets in the National Diet and Nutrition Study (NDNS), a representative UK sample. A food price database is linked to diet diary data to 
characterize individual-level diet costs, expressed as a daily diet cost. In addition, due to variability in individual energy requirements, costs are adjusted to $10 \mathrm{MJ}$ to enhance comparability. Furthermore, the sociodemographic data available for the sample enable the exploration of subgroup comparisons. Elucidating patterns in diet costs could have implications for the targeting of public health messages.

\section{Experimental methods}

\section{Sample and data collection}

The present cross-sectional study used data from the first two waves of the NDNS, collected in 2008-2010 ${ }^{(17)}$. The NDNS is a rolling national dietary monitoring programme, designed to track trends in dietary intake. In each year of data collection, a nationally representative sample of individuals is selected from private residences drawn from the Postcode Address File (PAF). In waves 1 and 2, $10 \%$ of the eligible addresses declined to take part before household selection. After selection, there was an overall response rate of $64 \%$ of households. The original sample comprised both children and adults; however, only adult data ( $\geq 19$ years; $n$ 1031) were included in the current analyses.

The NDNS assessed dietary intake using unweighed diaries on four consecutive days. Portion size photographs were included for fifteen commonly consumed foods; all other portions were estimated using household measures or package weights. Diary data were coded and recorded using the DINO (Diet In Nutrients Out) database.

Participant characteristics were ascertained by a trained interviewer in a face-to-face setting. These included: sex, age, educational qualifications, employment (NS-SEC8 (National Statistics Socio-economic Classification, eight categories)), marital status, household income (thirteen categories), household size and cigarette-smoking status (never, ex-regular, current regular). In addition, anthropometric data were measured by the interviewer. Achievement of the UK's ' 5 a day' recommendations for fruit and vegetable intake ('yes' or 'no') was calculated from the dietary data (including composite dishes) and was available as a variable in the NDNS data set. The ' 5 a day' criteria stipulate five portions, of $80 \mathrm{~g}$ each, of fruit and vegetables, including dried fruit $(30 \mathrm{~g} /$ portion) and up to one portion $(150 \mathrm{ml})$ of fruit juice, daily ${ }^{(18)}$. Energy intake was also presented within the NDNS data set, as calculated from diary data. Further details about recruitment, study design and data handling can be found in the survey report ${ }^{(19)}$.

In addition to the variables provided in the NDNS data set, four variables were derived for the purposes of the present study. One newly derived variable was a consequence of collapsing categories to facilitate statistical analyses: education was collapsed from seven to four categories (degree or higher education; GCE (General Certificate of Education) A-level or equivalent; GCSE (General Certificate of Secondary Education) or still in full-time education; no qualifications). Age, a continuous variable, was categorized into six bands: 19-29 years, 30-39 years, 40-49 years, 50-59 years, 60-69 years and 70 years or over. Equivalized income was derived from the midpoint of each category of household income, using the rescaled Organisation of Economic Co-operation and Development modified scale ${ }^{(20)}$, and categorized into five bands: up to £14999, £15000-£24999, $£ 25000-£ 34999$, £35000-£49999 and $\$ 50000$ or more per annum. Finally, alcohol consumption category was calculated from reported alcohol consumption in the diet diaries. The average daily quantity of alcohol consumed (grams) for each participant was converted to units ( 1 unit $=8 \mathrm{~g}$ ). Participants were then categorized according to national UK recommendations as: nonconsumers ( 0 units of alcohol consumed); low-risk consumers (up to an average of 3 units/d for women, 4 units/d for men); increasing-risk consumers (between 3 and 6 units/d for women, between 4 and 8 units/d for men); or higher-risk consumers ( 6 units and above/d for women, 8 units and above/d for men).

On examination of the data, it was evident that some participants had recorded diet for only three of the four data collection days. These individuals were excluded, reducing the available sample from 1031 to 1014.

\section{Food cost database}

To assign a cost for individuals' diets in the NDNS, it was necessary to assign a price to each food or beverage consumed. This was achieved by linking the NDNS data to a database of national food prices. The database used for this was created at the University of Leeds in 2004 and contains price information for over 3000 foods and drinks. Prices (lowest, mean and highest) from supermarket websites were calculated per $100 \mathrm{~g}$ (or $100 \mathrm{ml}$ ) edible weight, accounting for changes in weight associated with cooking and preparation where appropriate. Promotional or sale items were disregarded. Each food item is matched by code to the in-house dietary assessment tool, DANTE (Diet and Nutrition Tool for Evaluation), which utilizes nutrient information from the McCance and Widdowson food composition tables ${ }^{(21)}$.

\section{Diet cost calculation}

The food codes employed by DINO differ from those of DANTE. To assign a cost to the foods listed in the NDNS, it was necessary to create a look-up file to match the codes of each food. Both databases incorporate data from the UK food composition tables ${ }^{(21)}$ and, as such, it was possible to match many of the food items exactly (30\% of foods). Where an exact match for a food description was not available, the closest alternative was chosen. In the 
majority of cases, a close match was apparent - for example, 'peas boiled in salted water' could be matched to 'peas boiled in water'. If foods were coded as composite dishes in the NDNS (e.g. a ready meal lasagne), this was matched to the closest composite item in DANTE. The look-up file was created by the first author.

Once the data sets were matched, the mean database cost was applied to the quantity of food consumed by each participant and a daily average calculated:

Daily diet cost $(£ / d)=$

$\frac{\Sigma[\text { DANTE food price }(\text { pence } / \mathrm{g}) \times \text { quantity food consumed }(\mathrm{g})]}{\text { number of days }(4)} \div 100$.

Uncarbonated water was excluded from the daily diet cost calculation as it was not possible to distinguish from the data whether the water consumed was free tap water or purchased bottled water. Diet costs also exclude alcoholic beverages, due to the disproportionate influence of these expensive items on the diet costs of those who consume them. In addition, the price elasticity of demand reveals alcohol to be a complex commodity, perhaps suggesting that alcohol consumption is subject to different budgeting considerations to that of food and deserving of separate enquiry.

The database was populated using 2004 prices, whereas the NDNS data were collected between 2008 and 2010. Despite this, a correction for inflation was not applied in these analyses. This decision was taken on the grounds that the combined years of the NDNS data collection would make a correction for inflation unfeasible. In addition, it was felt that it is the relative costs within the population that are of interest for the purposes of the present study, as opposed to the absolute costs.

To adjust for differing energy requirements, costs were also calculated per $10 \mathrm{MJ}$. The value of $10 \mathrm{MJ}$ was selected as a midpoint between the Estimated Average Requirement for males and females (the Scientific Advisory Committee on Nutrition ${ }^{(22)}$ recommends an Estimated Average Requirement of $10.9 \mathrm{MJ} / \mathrm{d}$ for men and $8.7 \mathrm{MJ} / \mathrm{d}$ for women (adults aged 19 years or over)). The energyadjusted daily diet cost was calculated using the following formula:

Energy-adjusted cost $=\frac{\text { mean daily diet cost }(\mathfrak{f})}{\text { mean daily energy intake }(\mathrm{MJ})} \times 10$.

Outliers for both diet cost variables were identified; however, examination of the diary information did not reveal implausible dietary intakes. There were therefore no exclusions on this basis.

\section{Statistical analyses}

Both cost variables were positively skewed; therefore data were log transformed. Geometric mean and 95\% confidence intervals around the mean are presented.
Mean daily diet costs (£/d) and mean energy-adjusted costs $(£ / 10 \mathrm{MJ})$ were calculated for the whole sample and for each category of the following variables: age, sex, employment, qualifications, equivalized household income, household size, marital status, BMI classification, smoking status, alcohol consumption and ' 5 a day' achievement. Subgroup differences in daily and energyadjusted diet costs were tested using univariate regression analyses.

Multivariable regression models assessed the strength of each variable's relationship to diet costs (Model 1 examined daily diet costs and Model 2 energy-adjusted diet costs), adjusting for the other variables. Cost data were skewed, but residuals were normally distributed; therefore non-logged variables were used. All variables were included in the regression model. BMI was included as a continuous variable. Household income, as an ordinal variable, was also treated linearly. In addition, energy intake from food was included in the model with daily diet costs, but not in the model for costs per $10 \mathrm{MJ}$, because energy was used in the derivation of the latter variable. The underweight ( $n$ 13) were excluded from these analyses.

The NDNS sample weights were used to account for sampling probabilities and clustering. Details of the weights can be found in Appendix B of the survey report $^{(23)}$. In some sub-population analyses, strata occurred with singleton primary sample units. In these instances, standard errors were estimated using a centred correction.

Statistical analyses were performed using the SVY suite of commands in the statistical software package Stata IC release 12 . A two-way significance level of $5 \%$ was set.

\section{Ethical approval}

The present study contains secondary analyses of a national survey. The survey was conducted according to the guidelines laid down in the Declaration of Helsinki. Details on ethical approval can be found in the survey report ${ }^{(19)}$.

\section{Results}

The sample was $51 \%$ female and predominantly of white ethnic origin (91\%). Ages ranged from 19 to 94 years, with a roughly equal distribution across the age groups: $14-19 \%$ falling into each of the six categories. An equivalized household income of between $\$ 15000$ and $£ 24999$ per annum was most frequently reported ( $25 \%)$. Mean daily energy from food was 7408 (sD 2356) kJ. The geometric mean daily diet cost and energy-adjusted diet cost of the sample can be seen in Table 1 .

Unadjusted univariate regression analyses revealed significant differences between the categories of several sociodemographic variables (Table 1). These included 
Table 1 Geometric means and $95 \%$ confidence intervals for daily diet costs $(£ / \mathrm{d})$ and costs adjusted to $10 \mathrm{MJ}(£ / 10 \mathrm{MJ})$ for the weighted sample and subgroups; adults aged 19 years or over, UK National Diet and Nutrition Study, 2008-2010

\begin{tabular}{|c|c|c|c|c|c|c|c|}
\hline \multirow[b]{2}{*}{ Variable } & \multirow{2}{*}{$\begin{array}{l}\text { Weighted } \\
\text { sample size }\end{array}$} & \multicolumn{3}{|c|}{ Daily diet cost $(£ / d)$} & \multicolumn{3}{|c|}{ Energy-adjusted diet cost $(£ / 10 \mathrm{MJ})$} \\
\hline & & Mean & $95 \% \mathrm{Cl}$ & $P$ & Mean & $95 \% \mathrm{Cl}$ & $P$ \\
\hline Full sample & 1016 & $2 \cdot 89$ & $2 \cdot 81,2 \cdot 96$ & & $4 \cdot 09$ & $4 \cdot 01,4 \cdot 18$ & \\
\hline \multicolumn{8}{|l|}{ Sex } \\
\hline Male & 493 & $3 \cdot 15$ & $3 \cdot 01,3 \cdot 28$ & \multirow[t]{2}{*}{$<0.01$} & 3.91 & $3.79,4.03$ & \multirow[t]{2}{*}{$<0.01$} \\
\hline Female & 523 & $2 \cdot 66$ & $2 \cdot 58,2 \cdot 74$ & & $4 \cdot 28$ & $4 \cdot 17,4 \cdot 39$ & \\
\hline \multicolumn{8}{|l|}{ Age group } \\
\hline $19-29$ years & 195 & $2 \cdot 82$ & $2 \cdot 61,3.04$ & \multirow[t]{6}{*}{$0 \cdot 32$} & $3 \cdot 83$ & $3.63,4.04$ & \multirow[t]{6}{*}{0.03} \\
\hline $30-39$ years & 174 & 3.07 & $2 \cdot 90,3 \cdot 25$ & & $4 \cdot 12$ & $3 \cdot 95,4 \cdot 30$ & \\
\hline $40-49$ years & 194 & $2 \cdot 84$ & $2 \cdot 69,3 \cdot 00$ & & $4 \cdot 06$ & $3 \cdot 89,4 \cdot 24$ & \\
\hline $50-59$ years & 159 & $2 \cdot 97$ & $2 \cdot 81,3 \cdot 14$ & & $4 \cdot 25$ & $4 \cdot 09,4 \cdot 41$ & \\
\hline $60-69$ years & 141 & $2 \cdot 96$ & $2 \cdot 79,3 \cdot 14$ & & $4 \cdot 37$ & $4 \cdot 15,4 \cdot 60$ & \\
\hline 70 years or over & 152 & $2 \cdot 68$ & $2 \cdot 53,2 \cdot 84$ & & $4 \cdot 03$ & $3 \cdot 85,4 \cdot 72$ & \\
\hline \multicolumn{8}{|l|}{ Employment† } \\
\hline Higher managerial \& professional & 141 & $3 \cdot 42$ & $3 \cdot 19,3 \cdot 66$ & \multirow[t]{9}{*}{$<0.01$} & $4 \cdot 46$ & $4 \cdot 20,4 \cdot 72$ & \multirow[t]{9}{*}{$<0.01$} \\
\hline Lower managerial \& professional & 294 & 2.99 & $2 \cdot 86,3 \cdot 12$ & & $4 \cdot 30$ & $4 \cdot 16,4 \cdot 44$ & \\
\hline Intermediate occupations & 77 & $2 \cdot 90$ & $2 \cdot 69,3 \cdot 13$ & & $4 \cdot 29$ & $3.92,4.69$ & \\
\hline $\begin{array}{l}\text { Small employers \& own account } \\
\text { workers }\end{array}$ & 118 & $2 \cdot 96$ & $2 \cdot 78,3 \cdot 15$ & & $4 \cdot 15$ & $3 \cdot 87,4 \cdot 44$ & \\
\hline Lower technical \& supervisory & 112 & $2 \cdot 76$ & $2.58,2.95$ & & 3.95 & $3 \cdot 77,4 \cdot 13$ & \\
\hline Semi-routine occupations & 124 & $2 \cdot 46$ & $2 \cdot 29,2 \cdot 66$ & & $3 \cdot 65$ & $3 \cdot 49,3 \cdot 82$ & \\
\hline Routine occupations & 109 & $2 \cdot 67$ & $2 \cdot 39,2 \cdot 97$ & & $3 \cdot 67$ & $3 \cdot 47,3 \cdot 89$ & \\
\hline Never worked & 17 & $2 \cdot 57$ & $2 \cdot 13,3 \cdot 09$ & & $3 \cdot 80$ & $3 \cdot 41,4 \cdot 25$ & \\
\hline Other & 25 & $2 \cdot 64$ & $2 \cdot 15,3 \cdot 25$ & & $4 \cdot 02$ & $3.52,4.58$ & \\
\hline \multicolumn{8}{|l|}{ Marital status } \\
\hline Single, never married & 307 & $2 \cdot 87$ & $2 \cdot 71,3.05$ & 0.02 & $3 \cdot 92$ & $3 \cdot 79,4 \cdot 08$ & 0.07 \\
\hline Married & 530 & $2 \cdot 96$ & $2 \cdot 87,3.06$ & & $4 \cdot 15$ & $4 \cdot 05,4 \cdot 26$ & \\
\hline Married but separated & 19 & $2 \cdot 84$ & $2 \cdot 34,3 \cdot 46$ & & $4 \cdot 45$ & $3.99,4.96$ & \\
\hline Divorced & 87 & $2 \cdot 77$ & $2 \cdot 57,2 \cdot 98$ & & $4 \cdot 26$ & $4 \cdot 01,4 \cdot 52$ & \\
\hline Widowed & 73 & $2 \cdot 55$ & $2 \cdot 34,2 \cdot 78$ & & $4 \cdot 11$ & $3 \cdot 86,4 \cdot 38$ & \\
\hline Qualificationsł & & & & & & & \\
\hline Degree or higher education & 345 & $3 \cdot 20$ & $3.08,3.34$ & $<0.01$ & $4 \cdot 32$ & $4 \cdot 20,4 \cdot 44$ & $<0.01$ \\
\hline GCE A-level or equivalent, foreign & 173 & $2 \cdot 97$ & $2 \cdot 84,3 \cdot 11$ & & $4 \cdot 07$ & $3.93,4 \cdot 23$ & \\
\hline GCSE or still in full-time education & 257 & $2 \cdot 79$ & $2 \cdot 64,2 \cdot 95$ & & $4 \cdot 07$ & $3 \cdot 88,4 \cdot 26$ & \\
\hline No qualifications & 234 & $2 \cdot 51$ & $2 \cdot 37,2 \cdot 67$ & & $3 \cdot 81$ & $3 \cdot 67,3.95$ & \\
\hline $\begin{array}{l}\text { Equivalized household income (per } \\
\text { annum) }\end{array}$ & & & & & & & \\
\hline Up to $£ 14999$ & 189 & $2 \cdot 55$ & $2 \cdot 42,2 \cdot 68$ & $<0.01$ & 3.69 & $3.55,3.84$ & $<0.01$ \\
\hline$£ 15000-£ 24999$ & 217 & $2 \cdot 77$ & $2 \cdot 64,2 \cdot 91$ & & 3.99 & $3 \cdot 84,4 \cdot 14$ & \\
\hline$£ 25000-£ 34999$ & 179 & $2 \cdot 87$ & $2 \cdot 72,3.02$ & & $4 \cdot 16$ & $3 \cdot 97,4 \cdot 36$ & \\
\hline$£ 35000-£ 49999$ & 138 & $3 \cdot 21$ & $3.02,3 \cdot 42$ & & $4 \cdot 32$ & $4 \cdot 13,4 \cdot 51$ & \\
\hline$£ 50000$ or more & 133 & 3.37 & $3 \cdot 15,3 \cdot 60$ & & $4 \cdot 58$ & $4 \cdot 35,4 \cdot 82$ & \\
\hline Household size & & & & & & & \\
\hline 1 person & 169 & $2 \cdot 77$ & $2 \cdot 63,2 \cdot 91$ & $0 \cdot 70$ & $4 \cdot 10$ & $3 \cdot 94,4 \cdot 29$ & 0.02 \\
\hline 2 people & 355 & $2 \cdot 96$ & $2 \cdot 82,3 \cdot 10$ & & $4 \cdot 27$ & $4 \cdot 12,4 \cdot 43$ & \\
\hline 3 or 4 people & 371 & $2 \cdot 88$ & $2 \cdot 76,3 \cdot 01$ & & 3.96 & $3 \cdot 86,4 \cdot 07$ & \\
\hline 5 or more people & 121 & $2 \cdot 85$ & $2 \cdot 67,3 \cdot 05$ & & 3.97 & $3 \cdot 75,4 \cdot 20$ & \\
\hline BMI category & & & & & & & \\
\hline Underweight $\left(<18.5 \mathrm{~kg} / \mathrm{m}^{2}\right)$ & 12 & $2 \cdot 21$ & $1 \cdot 80,2 \cdot 72$ & $0 \cdot 17 \S$ & $3 \cdot 23$ & $2 \cdot 69,3 \cdot 87$ & $0.25 \S$ \\
\hline Normal weight $\left(18.5-24.9 \mathrm{~kg} / \mathrm{m}^{2}\right)$ & 323 & $2 \cdot 94$ & $2 \cdot 78,3 \cdot 10$ & & $4 \cdot 01$ & $3 \cdot 87,4 \cdot 16$ & \\
\hline Overweight $\left(25 \cdot 0-29 \cdot 9 \mathrm{~kg} / \mathrm{m}^{2}\right)$ & 346 & $2 \cdot 99$ & $2 \cdot 87,3 \cdot 11$ & & $4 \cdot 15$ & $4 \cdot 01,4 \cdot 28$ & \\
\hline Obese $\left(\geq 30 \mathrm{~kg} / \mathrm{m}^{2}\right)$ & 259 & $2 \cdot 78$ & $2 \cdot 64,2 \cdot 93$ & & $4 \cdot 13$ & $3 \cdot 98,4 \cdot 28$ & \\
\hline Smoking & & & & & & & \\
\hline Never smoked & 553 & $2 \cdot 95$ & $2 \cdot 84,3 \cdot 06$ & $<0 \cdot 01$ & $4 \cdot 15$ & $3 \cdot 67,4 \cdot 00$ & $<0.01$ \\
\hline Ex-smoker & 245 & $2 \cdot 97$ & $2 \cdot 83,3 \cdot 13$ & & $4 \cdot 20$ & $4 \cdot 06,4 \cdot 35$ & \\
\hline Current smoker & 217 & $2 \cdot 63$ & $2 \cdot 48,2 \cdot 79$ & & $3 \cdot 83$ & $4 \cdot 04,4 \cdot 26$ & \\
\hline Alcohol consumption & & & & & & & \\
\hline None & 408 & $2 \cdot 58$ & $2 \cdot 48,2 \cdot 69$ & $<0.01$ & 3.93 & $3 \cdot 82,4 \cdot 04$ & $<0.05$ \\
\hline Low risk & 420 & 3.05 & $2 \cdot 94,3 \cdot 17$ & & $4 \cdot 22$ & $4 \cdot 10,4 \cdot 35$ & \\
\hline Increasing risk & 135 & $3 \cdot 19$ & $2 \cdot 99,3 \cdot 41$ & & $4 \cdot 29$ & $4.05,4.55$ & \\
\hline High risk & 53 & 3.34 & $3 \cdot 02,3 \cdot 70$ & & 3.90 & $3.57,4.25$ & \\
\hline Achieve ' 5 a day' & & & & & & & \\
\hline Yes & 325 & 3.48 & $3 \cdot 35,3 \cdot 62$ & $<0.01$ & $4 \cdot 55$ & $4 \cdot 40,4 \cdot 71$ & $<0.01$ \\
\hline No & 690 & $2 \cdot 64$ & $2 \cdot 56,2 \cdot 72$ & & $3 \cdot 89$ & $3 \cdot 80,4 \cdot 71$ & \\
\hline
\end{tabular}

*Weighted population numbers are rounded to the nearest whole unit.

+More details about the NS-SEC8 (National Statistics Socio-economic Classification, eight categories) occupation classification scheme can be found at http:// www.ons.gov.uk/ons/guide-method/.classifications/current-standard-classifications/soc2010/soc2010-volume-3-ns-sec-rebased-on-soc2010-user-manual/ index.html.

fUK qualifications: GCSE (General Certificate of Secondary Education) typically taken when students are aged 14-16 years; GCE (General Certificate of Education) A-levels taken pre-university or for completion of secondary school; 'degree or higher education' refers to post-secondary school, or tertiary, qualifications.

$\S$ Excluding underweight. 
Table 2 Regression of sociodemographic and lifestyle variables on estimates of daily diet cost (Model 1) and costs per 10 MJ (Model 2); adults aged 19 years or over, UK National Diet and Nutrition Study, 2008-2010 ( $n$ 808)

\begin{tabular}{|c|c|c|c|c|c|c|}
\hline \multirow[b]{2}{*}{ Variable } & \multicolumn{3}{|c|}{ Model 1: daily diet cost (pence/d) } & \multicolumn{3}{|c|}{ Model 2: costs per $10 \mathrm{MJ}$ (pence/10 MJ) } \\
\hline & $\begin{array}{c}\text { Coefficient } \\
\text { (difference in } \\
\text { diet cost, pence) }\end{array}$ & $95 \% \mathrm{Cl}$ & $\begin{array}{l}\text { Overall } \\
P \text { value }\end{array}$ & $\begin{array}{c}\text { Coefficient } \\
\text { (difference in } \\
\text { diet cost, pence) }\end{array}$ & $95 \% \mathrm{Cl}$ & $\begin{array}{l}\text { Overall } \\
P \text { value }\end{array}$ \\
\hline $\operatorname{Sex}^{*}$ & $8 \cdot 33$ & $-5 \cdot 20,21 \cdot 85$ & 0.973 & $39 \cdot 23$ & $19 \cdot 36,59 \cdot 09$ & $<0.001$ \\
\hline Age group & 0.08 & $-4 \cdot 91,5 \cdot 08$ & 0.973 & $1 \cdot 68$ & $-8 \cdot 37,11 \cdot 72$ & 0.742 \\
\hline Food energy (kJ) & 0.03 & $0.03,0.03$ & $<0.001$ & - & - & - \\
\hline BMI $\left(\mathrm{kg} / \mathrm{m}^{2}\right)$ & 1.09 & $0 \cdot 02,2 \cdot 16$ & 0.046 & $1 \cdot 96$ & $0.41,3.51$ & 0.013 \\
\hline Cigarette-smoking statust & & & $0 \cdot 841$ & & & 0.985 \\
\hline Current regular smoker & $-5 \cdot 23$ & $-23 \cdot 15,12 \cdot 70$ & & $2 \cdot 07$ & $-26 \cdot 41,30 \cdot 55$ & \\
\hline Ex-regular smoker & -1.93 & $-13 \cdot 85,9 \cdot 98$ & & $1 \cdot 24$ & $-16 \cdot 57,19 \cdot 05$ & \\
\hline Achieve ' 5 a day' & $37 \cdot 87$ & $25 \cdot 13,50 \cdot 61$ & $<0.001$ & $48 \cdot 86$ & $26 \cdot 88,70 \cdot 83$ & $<0.001$ \\
\hline Household income group & $10 \cdot 50$ & $5 \cdot 78,15 \cdot 21$ & $<0.001$ & $13 \cdot 73$ & $6 \cdot 80,20 \cdot 65$ & $<0.001$ \\
\hline Marital status $\S$ & & & 0.604 & & & 0.603 \\
\hline Married & $2 \cdot 20$ & $-12 \cdot 88,17 \cdot 29$ & & $3 \cdot 13$ & $-18 \cdot 63,24 \cdot 89$ & \\
\hline Married but separated & $42 \cdot 58$ & $-8 \cdot 13,93 \cdot 30$ & & 63.04 & $-13.02,139 \cdot 09$ & \\
\hline Divorced & $1 \cdot 93$ & $-20 \cdot 64,24 \cdot 50$ & & $11 \cdot 60$ & $-27 \cdot 30,50 \cdot 51$ & \\
\hline Widowed & $4 \cdot 65$ & $-20 \cdot 84,30 \cdot 14$ & & $5 \cdot 48$ & $-34 \cdot 51,45 \cdot 48$ & \\
\hline Qualificationsll & & & 0.003 & & & 0.086 \\
\hline GCSE or still in full-time education & $18 \cdot 63$ & $1 \cdot 03,36 \cdot 23$ & & $27 \cdot 06$ & $-0 \cdot 64,54 \cdot 76$ & \\
\hline GCE A-level or equivalent & $12 \cdot 00$ & $-5 \cdot 23,29 \cdot 24$ & & $9 \cdot 57$ & $-18 \cdot 34,37 \cdot 49$ & \\
\hline Degree or equivalent & $25 \cdot 88$ & $9 \cdot 07,42 \cdot 69$ & & $38 \cdot 49$ & $5 \cdot 27,71 \cdot 72$ & \\
\hline Household size & $-7 \cdot 38$ & $-15 \cdot 07,0 \cdot 31$ & 0.060 & $-12 \cdot 40$ & $-26 \cdot 15,1 \cdot 35$ & 0.077 \\
\hline NS-SEC8 classification & & & 0.048 & & & $0 \cdot 101$ \\
\hline Lower managerial \& professional & $-14 \cdot 36$ & $-38 \cdot 10,9 \cdot 37$ & & $-18 \cdot 60$ & $-54 \cdot 23,17 \cdot 03$ & \\
\hline Intermediate occupations & $-10 \cdot 76$ & $-39 \cdot 59,18 \cdot 07$ & & -4.91 & $-65 \cdot 87,56 \cdot 04$ & \\
\hline Small employers \& own account workers & $-7 \cdot 06$ & $-38 \cdot 02,23 \cdot 89$ & & $-8 \cdot 30$ & $-56 \cdot 05,39 \cdot 46$ & \\
\hline Lower technical \& supervisory & $-14 \cdot 24$ & $-38 \cdot 23,9 \cdot 75$ & & $-25 \cdot 15$ & $-63 \cdot 70,13 \cdot 40$ & \\
\hline Semi-routine occupations & $-33 \cdot 88$ & $-57 \cdot 92,-9 \cdot 85$ & & $-48 \cdot 61$ & $-84 \cdot 67,-12 \cdot 54$ & \\
\hline Routine occupations & $-18 \cdot 89$ & $-51 \cdot 70,13 \cdot 92$ & & $-41 \cdot 49$ & $-83 \cdot 23,0 \cdot 26$ & \\
\hline Never worked & -21.91 & $-71 \cdot 08,27 \cdot 25$ & & $-27 \cdot 03$ & $-103 \cdot 62,49 \cdot 55$ & \\
\hline Other & -19.03 & $-67 \cdot 39,29 \cdot 34$ & & $-46 \cdot 36$ & $-113 \cdot 17,20 \cdot 45$ & \\
\hline Alcohol consumption group & $8 \cdot 26$ & $0.61,15.92$ & 0.035 & $6 \cdot 46$ & $-3 \cdot 82,16 \cdot 75$ & 0.216 \\
\hline
\end{tabular}

GCSE, General Certificate of Secondary Education; GCE, General Certificate of Education; NS-SEC8, National Statistics Socio-economic Classification, eight categories.

Underweight participants $\left(\mathrm{BMl}<18.5 \mathrm{~kg} / \mathrm{m}^{2}\right)$ excluded.

${ }^{*}$ Reference category $=$ males.

tCompared with participants who have never regularly smoked (reference category).

‡Equivalized household income categories: up to £14999; £15000-£24 999; £25 000-£34 999; £35000-£49999; £50 000 or more per annum.

§Reference category $=$ single, never married.

IICompared with participants with no qualifications.

TReference category = higher managerial \& professional.

sex, employment, qualifications, marital status and household income. In addition, significant differences were apparent for the lifestyle variables: cigarette smoking; consumption of alcohol; and consumption of fruit and vegetables (five portions daily). With the exception of marital status, all of these differences remained after adjusting costs to $10 \mathrm{MJ}$. In addition, significant differences in costs per $10 \mathrm{MJ}$ were apparent between categories of age and household size. Diet costs were not found to differ significantly between categories of BMI.

In the multivariable regression analyses, data were missing for 193 participants, leaving an analytical sample of 808. Missing data for income ( $n$ 137) and BMI ( $n$ 76) accounted for the majority of dropped observations. Participants with missing data were more likely to be from the oldest age category. Diet costs were similar to those in the full sample.

Model 1 results indicated that daily diet costs were significantly greater with higher energy intake, after adjusting for the other variables (see Table 2): each additional $400 \mathrm{~kJ}$ (approximately $100 \mathrm{kcal}$ ) was associated with an extra 12 pence. Achieving ' 5 a day' was associated with an extra 38 pence. There was also a significant overall effect of equivalized household income category on daily diet costs, with an additional 10 pence associated with each progression up through the categories (the model-estimated cost at the lowest income category was £2.90). A significant overall effect was apparent for qualifications and employment classification. In addition, BMI was positively associated with diet costs.

Table 2 also presents the results of Model 2. This model revealed significant effects of household income and achieving ' 5 a day', as was found in Model 1. Furthermore, in Model 2, each higher income category was associated with an additional 14 pence per $10 \mathrm{MJ}$, and those who achieved ' 5 a day' had an energy-adjusted cost of 49 pence more per $10 \mathrm{MJ}$ than those who did not. For BMI, each additional $\mathrm{kg} / \mathrm{m}^{2}$ was associated with an 
additional 2 pence per $10 \mathrm{MJ}$. In contrast to the first model, however, a significant effect was observed for sex, with females showing costs of 39 pence per $10 \mathrm{MJ}$ higher than males, while estimates for qualifications and employment did not achieve significance.

After adjustment, no significant effects were apparent in either model for age group or cigarette-smoking status.

\section{Discussion}

This is the first time that a monetary value has been applied to individuals' diets in the NDNS. These costs are estimates of the inherent value of diets, as opposed to actual expenditure. For this reason, as well as the fact that the price data were collected in a different year from the dietary data, the diet costs presented here will not be directly comparable to the findings of food expenditure studies. Instead, the findings contribute to our understanding of patterns in the inherent monetary value of diets across sociodemographic variables.

The monetary value of diets was strongly associated with energy intake $(r=0 \cdot 66)$, indicating that those with higher energy requirements face higher diet costs. Due to this relationship, adjusting diet costs to $10 \mathrm{MJ}$ should allow a fairer comparison.

Univariate comparisons highlighted interesting differences between subgroups in this sample. For example, men were estimated to have higher daily diet costs than women in this sample, but lower diet costs per $10 \mathrm{MJ}$. This is a pattern similarly reported in a French ${ }^{(24)}$ and a $\mathrm{US}^{(10)}$ sample, although not apparent in all studies of this type $^{(9)}$. The pattern likely reflects the higher energy intakes that tend to be observed in males, with diet costs and energy intakes being strongly correlated. After adjusting costs to $10 \mathrm{MJ}$, males exhibited lower costs, probably as a result of having more energy-dense diets, a sex difference similarly reported in other samples ${ }^{(25,26)}$. In the multivariable analysis, however, sex no longer had a significant effect on daily diet costs. This supports the explanation above, because the inclusion of energy intake as a covariate for daily diet costs resulted in a loss of statistical significance. However, a difference between the sexes was still apparent when diet cost per $10 \mathrm{MJ}$ was the outcome.

Both diet cost variables were found to increase monotonically with income categories in this sample. The increase in cost per $10 \mathrm{MJ}$ with rising income categories is particularly interesting: because the food price database uses mean values, it implies that the additional costs incurred by the higher income categories result from the selection of different foods, rather than 'trading up' to higher-quality, more expensive versions of the same items. In reality, higher-income participants may also have 'traded up' in addition to choosing different foods from lower-income subjects, which would augment the observed diet cost differences. Similar income effects have been observed in some ${ }^{(6,9)}$, although not all ${ }^{(12)}$, comparable studies. (The authors of the latter study suggest the lack of significance may be attributed to a lack of statistical power in the sample, or inappropriate income measurement.)

Those in managerial and professional positions showed higher diet costs than other occupations; as did those with higher compared with lower educational qualifications. Differences in diet cost by education have been described in other countries ${ }^{(6,9,10)}$, but previous studies have not reported occupation differences in diet costs. The influence of education and occupation on diet costs could be indirect, through links with income. Alternatively, diet selection may be influenced by education and occupation independently. In the literature, education appears to be more strongly associated with dietary habits than occupation ${ }^{(27,28)}$, although one study implies that the effect of education may be mediated by the influence of income ${ }^{(29)}$.

Significant associations with daily diet cost were evident for each of these socio-economic indicators (income, qualifications and employment) after adjusting for the other variables in the regression analysis. This supports the suggestion that they are independently influential. However, only income was significantly associated with diet costs per $10 \mathrm{MJ}$.

Differences in diet costs per $10 \mathrm{MJ}$ were evident between smokers and non-smokers in the current study. It could be speculated from this relationship that the monetary costs of smoking impinge upon the food budget. Conversely, the findings may reflect a clustering of behaviours (smoking and poor diet). The latter interpretation is supported by the observation that cigarettesmoking status was not found to be significantly related to diet costs after adjusting for other variables. In other populations, comparisons between smokers and nonsmokers have resulted in mixed findings ${ }^{(14,15)}$.

In this sample, the observation of increasing daily diet costs with increasing alcohol consumption could also be attributed to the concomitant increasing intakes of food energy (not presented). However, those who consumed no alcohol exhibited a similar median cost to the highest alcohol consumers when adjusted to $10 \mathrm{MJ}$, suggesting that the observed differences are not solely due to energy differences and again supporting a behaviour-cluster interpretation. A previous study ${ }^{(30)}$ has identified a significant pattern of lower diet quality with increasing alcohol consumption, but only a few have reported increasing food energy intake ${ }^{(31)}$ or a tendency to overreport food intake among higher alcohol consumers ${ }^{(32)}$. On the other hand, it is also possible that drinking behaviours are linked to disposable incomes and thereby affect food budgets.

Both daily diet costs and diet costs per $10 \mathrm{MJ}$ were positively associated with BMI in the multivariable regression 
analyses, indicating higher costs with increasing body mass. This finding contrasts with the negative association between BMI and diet cost found in a cross-sectional survey of Japanese students ${ }^{(15)}$, but is in keeping with a longitudinal study in Spain which indicated increased odds of weight gain among those who had higher diet costs at baseline ${ }^{(14)}$. The apparent positive relationship between diet costs and BMI does not lend support to the idea that food prices have contributed to obesity rates ${ }^{(6,7)}$. However, the limitations of the current cross-sectional study (see below) do not allow a causal interpretation, and this aspect warrants further investigation.

Diets containing five portions or more of fruit and vegetables daily were found to be of higher monetary value than those that featured fewer. This supports the findings of previous research suggesting that people who score more favourably on healthy diet indicators $^{(7,12,13,16,24,33)}$, as well as those who consume more fruit and vegetables in particular ${ }^{(9)}$, tend to spend more money on food or consume higher-value diets. In addition, the findings presented here go further than many of the other studies in showing that the relationship between fruit and vegetable consumption and diet costs remains even after adjusting for other economic and demographic factors. While some studies report that a diet adhering to national guidelines is theoretically achievable on low incomes (e.g. in the USA ${ }^{(34)}$ ), others have found that modelling diets to be both palatable and nutritionally adequate does increase costs ${ }^{(35)}$. One study in Ireland predicted that the cost of adhering to proposed guidelines, while achievable in theory, would take up to $100 \%$ of the income from welfare for an adolescent male ${ }^{(36)}$.

The current study did not investigate costs according to wider measures of diet quality nor adherence to guidelines other than fruit and vegetables. Nevertheless, the results imply that the better-quality diets, as signified by the consumption of fruit and vegetables, were of higher intrinsic monetary worth. It cannot be determined from this study design whether diet costs were influential in participants' food selection; nevertheless, the relationships evident between diet costs and socio-economic markers are interesting, with potential policy implications, especially if fiscal interventions are being considered.

\section{Limitations}

Assigning costs to dietary data using a food price database is a potentially useful methodology. It is not without limitations, however. First, it should be noted that these diet cost estimates will inevitably echo any measurement error associated with the dietary assessment tool from which they are extrapolated. Under-reporting of food consumption, for example, will result in an underestimation of diet cost. Where under-reporting may be more prevalent among certain subgroups, as it has been suggested to be for those classified as obese for example ${ }^{(37)}$, the resulting bias could influence the results of subgroup comparisons. In this sample, energy intake was found to vary significantly between BMI categories, with the lowest energy intake reported in the obese (not presented). This perhaps suggests that such bias exists within the sample. Unfortunately, the NDNS does not contain physical activity data for the main sample, making it problematic to evaluate the extent of under-reporting. Other forms of measurement error associated with diet diaries could also have biased diet cost estimations, including dietary behaviour change in response to the assessment ${ }^{(38)}$.

This method of costing has limits in establishing the role of diet costs in food selection. First, because the results imply that the diets of certain subgroups are worth more, not necessarily that these populations spend more on their diets. The value of a person's diet may not reflect the prices he/she encountered in purchasing his/her foods: the food cost database does not account for restaurant or takeaway meals, for example, which are likely to be higher than those estimated; nor can it identify free, shared or foraged food. Second, as a crosssectional study, it is impossible to gauge whether diets of a lower monetary value are selected as a result of budgetary considerations or whether the value of a diet merely reflects a preference for cheaper foods due to other factors.

\section{Strengths}

These findings add to the literature on social inequalities in diet and health. Many of the patterns revealed here appear to substantiate speculated differences in diet costs, which should impart confidence to the costing method.

The existence of diet cost differences between certain groups of people could have implications in the consideration of proposed fiscal interventions to combat public health issues such as obesity (as suggested in one recent report ${ }^{(39)}$ ), that may differentially affect socioeconomic groups. This is concerning, especially given that the differences between sociodemographic groups observed here are likely to be conservative.

Individual-level diet costs will allow the investigation of diet costs in relation to health outcomes. Therefore, the present study paves the way for further investigations linking the monetary value of diets in the UK with dietary quality and ultimately health. Further investigations of this kind are planned in this sample.

\section{Conclusions}

The current study quantified individual diet costs for a representative UK sample. The findings suggest that certain subgroups in the UK consume diets of lower monetary value - those in the lower income categories and those who do not consume the recommended 
quantity of fruit and vegetables, for example. Costing diets in this manner is constrained by the measurement error associated with dietary assessment. Nevertheless, further research is now possible investigating the links between diet costs and health in a representative UK sample.

\section{Acknowledgements}

Sources of funding: K.A.T. is funded by an Economic and Social Research Council/Medical Research Council (ESRC/MRC) PhD studentship. ESRC and MRC had no role in the design, analysis or writing of this article. The original data creators, depositors or copyright holders of the NDNS and the UK Data Archive bear no responsibility for their further analysis or interpretation. NDNS copyright: Crown copyright is held jointly with the National Centre for Social Research. Crown copyright material is reproduced with the permission of the Controller of HMSO and the Queen's Printer for Scotland. Conflicts of interest: There are no conflicts of interest to declare. Ethical approval: This study contains secondary analyses of the NDNS. The NDNS obtained ethical approval from the Oxfordshire A Research Ethics Committee. Further details of ethical approval are described in the survey report ${ }^{(19)}$. Authors' contributions: K.A.T. carried out the analysis and wrote the first draft of this paper. J.E.C. and C.H. provided guidance through $\mathrm{PhD}$ supervision, as well as comments and edits on the paper drafts. Acknowledgements: The authors would like to acknowledge Kevin Tarbutt (funded by a Rank Prize Fund) and Edmund Parks, who created and updated the DANTE cost database, as well as the original NDNS data creators, depositors and funders: the National Centre for Social Research, the Northern Ireland Statistics and Research Agency, the Medical Research Council, University College London Medical School, the Food Standards Agency, the Department of Health and the UK Data Archive.

\section{References}

1. Steptoe A, Pollard TM \& Wardle J (1995) Development of a measure of the motives underlying the selection of food: the food choice questionnaire. Appetite 25, 267-284.

2. Connors M, Bisogni CA, Sobal J et al. (2001) Managing values in personal food systems. Appetite 36, 189-200.

3. Shepherd J, Harden A, Rees R et al. (2006) Young people and healthy eating: a systematic review of research on barriers and facilitators. Health Educ Res 21, 239-257.

4. Scheibehenne B, Miesler L \& Todd P (2007) Fast and frugal food choices: uncovering individual decision heuristics. Appetite 49, 578-589.

5. Nelson M, Erens B, Bates B et al. (2007) Low Income Diet and Nutrition Survey. London: TSO.

6. Monsivais P, McLain J \& Drewnowski A (2010) The rising disparity in the price of healthful foods: 2004-2008. Food Policy 35, 514-520.

7. Darmon N, Briend A \& Drewnowski A (2004) Energydense diets are associated with lower diet costs: a community study of French adults. Public Health Nutr 7, 21-27.
8. Department for Environment, Food and Rural Affairs (2004) Family Food: A Report on the 2003-04 Expenditure and Food Survey. London: Office for National Statistics \& Defra; available from http://www.defra.gov.uk/statistics/ foodfarm/food/familyfood/

9. Rehm CD, Monsivais P \& Drewnowski A (2011) The quality and monetary value of diets consumed by adults in the United States. Am J Clin Nutr 94, 1333-1339.

10. Monsivais P \& Drewnowski A (2009) Lower-energy-density diets are associated with higher monetary costs per kilocalorie and are consumed by women of higher socioeconomic status. J Am Diet Assoc 109, 814-822.

11. Maillot M, Darmon N, Darmon M et al. (2007) Nutrientdense food groups have high energy costs: an econometric approach to nutrient profiling. J Nutr 137, 1815-1820.

12. Waterlander WE, de Haas WE, van Amstel I et al. (2010) Energy density, energy costs and income - how are they related? Public Health Nutr 13, 1599-1608.

13. Schroder H, Marrugat J \& Covas MI (2006) High monetary costs of dietary patterns associated with lower body mass index: a population-based study. Int J Obes (Lond) 30, 1574-1579.

14. Lopez CN, Martinez-Gonzalez MA, Sanchez-Villegas A et al. (2009) Costs of Mediterranean and western dietary patterns in a Spanish cohort and their relationship with prospective weight change. J Epidemiol Community Health 63, 920-927.

15. Murakami K, Sasaki S, Okubo H et al. (2007) Monetary costs of dietary energy reported by young Japanese women: association with food and nutrient intake and body mass index. Public Health Nutr 10, 1430-1439.

16. Cade J, Upmeier H, Calvert C et al. (1999) Costs of a healthy diet: analysis from the UK Women's Cohort Study. Public Health Nutr 2, 505-512.

17. National Centre for Social Research, Medical Research Council, Resource Centre for Human Nutrition Research et al. (2010) National Diet and Nutrition Survey, 2008-2010, 3rd ed. Colchester: UK Data Archive.

18. Cullum A (2003) Increasing fruit and vegetable consumption: the 5 A DAY programme. Nutr Bull 28, 159-163.

19. Bates B, Lennox A, Bates C et al. (2011) National Diet and Nutrition Survey, Headline results from Years 1 and 2 (combined) of the Rolling Programme (2008/20092009/2010). https://www.gov.uk/government/uploads/ system/uploads/attachment_data/file/216484/dh_128550.pdf (accessed October 2013).

20. Anyaegbu G (2010) Using the OECD equivalence scale in taxes and benefits analysis. Econ Labour Mark Rev 4, 49-54.

21. Food Standards Agency (2002) McCance and Widdowson's The Composition of Foods, Sixth summary edition. Cambridge: Royal Society of Chemistry.

22. Scientific Advisory Committee on Nutrition (2011) Dietary Reference Values for Energy. London: TSO.

23. Department of Health (2012) National Diet and Nutrition Survey: Headline Results from Years 1, 2 and 3 (combined) of the Rolling Programme 2008/09-2010/11. http:// webarchive.nationalarchives.gov.uk/20130402145952/ http://transparency.dh.gov.uk/2012/07/25/ndns-3-yearsreport/ (accessed June 2013).

24. Maillot M, Darmon N, Vieux F et al. (2007) Low energy density and high nutritional quality are each associated with higher diet costs in French adults. Am J Clin Nutr 86, 690-696.

25. Ledikwe JH, Blanck HM, Khan LK et al. (2005) Dietary energy density determined by eight calculation methods in a nationally representative United States population. J Nutr 135, 273-278.

26. Marti-Henneberg C, Capdevila F, Arija V et al. (1999) Energy density of the diet, food volume and energy intake 
by age and sex in a healthy population. Eur J Clin Nutr $\mathbf{5 3}$, 421-428.

27. De Irala-Estevez J, Groth M, Johansson L et al. (2000) A systematic review of socio-economic differences in food habits in Europe: consumption of fruit and vegetables. Eur J Clin Nutr 54, 706-714.

28. Giskes K, Avendano M, Brug J et al. (2010) A systematic review of studies on socioeconomic inequalities in dietary intakes associated with weight gain and overweight/ obesity conducted among European adults. Obes Rev 11, 413-429.

29. Lallukka T, Pitkaniemi J, Rahkonen O et al. (2010) The association of income with fresh fruit and vegetable consumption at different levels of education. Eur J Clin Nutr 64, 324-327.

30. Breslow RA, Guenther PM \& Smothers BA (2006) Alcohol drinking patterns and diet quality: the 1999-2000 National Health and Nutrition Examination Survey. Am J Epidemiol 163, 359-366.

31. Kesse E, Clavel-Chapelon F, Slimani N et al. (2001) Do eating habits differ according to alcohol consumption? Results of a study of the French cohort of the European Prospective Investigation into Cancer and Nutrition (E3N-EPIC). Am J Clin Nutr 74, 322-327.

32. Zhang J, Temme EHM \& Kesteloot H (2001) Alcohol drinkers overreport their energy intake in the BIRNH
Study: evaluation by 24-hour urinary excretion of cations. J Am College Nutr 20, 510-519.

33. Ryden P \& Hagfors L (2011) Diet cost, diet quality and socio-economic position: how are they related and what contributes to differences in diet costs? Public Health Nutr 14, 1680-1692.

34. Cassady D, Jetter K \& Culp J (2007) Is price a barrier to eating more fruits and vegetables for low-income families? J Am Diet Assoc 107, 1909-1915.

35. Darmon N, Ferguson EL \& Briend A (2006) Impact of a cost constraint on nutritionally adequate food choices for French women: an analysis by linear programming. J Nutr Educ Behav 38, 82-90.

36. Flynn MAT, O'Brien CM, Ross V et al. (2012) Revision of food-based dietary guidelines for Ireland, Phase 2: recommendations for healthy eating and affordability. Public Health Nutr 15, 527-537.

37. Rennie KL, Coward A \& Jebb SA (2007) Estimating underreporting of energy intake in dietary surveys using an individualised method. Br J Nutr 97, 1169-1176.

38. Vuckovic N, Ritenbaugh C, Taren DL et al. (2000) A qualitative study of participants' experiences with dietary assessment. J Am Diet Assoc 100, 1023-1028.

39. Sustain (2013) A Children's Future Fund: How food duties could provide the money to protect children's bealth and the world they grow up in. London: Sustain. 